\title{
STABILITY AND ZERO-HOPF BIFURCATION ANALYSIS OF A TUMOUR AND T-HELPER CELLS INTERACTION MODEL IN THE CASE OF HIV INFECTION
}

\author{
GAMZEGUL KARAHISARLI, HUSEYIN MERDAN, AND ABDESSAMAD TRIDANE
}

Received 07 September, 2020

\begin{abstract}
In this paper, we present a mathematical model governing the dynamics of tumourimmune cells interaction under HIV infection. The interactions between tumour cells, helper T-cells, infected helper T-cells and virus cells are explained by using delay differential equations including two different discrete time delays. In the model, these time lags describe the time needed by the helper T-cells to find (or recognize) tumour cells and virus, respectively. First, we analyze the dynamics of the model without delays. We prove the positivity of the solution, analyze the local and global stabilities of the steady states of the model. Second, we study the effects of two discrete time delays on the stability of the endemically infected equilibrium point. We determine the conditions on parameters at which the system undergoes a zero-Hopf bifurcation. Choosing one of the delay terms as a bifurcation parameter and fixing the other, we show that a zero-Hopf bifurcation arises as the bifurcation parameter passes through a critical value. Finally, we perform numerical simulations to support and extend our theoretical results. The results concluded help to better understand the links between the immune system and the tumour development in the case of HIV infection.
\end{abstract}

2010 Mathematics Subject Classification: 34D20; 34D23; 34A34

Keywords: HIV infection, tumour, T-helper cells, delay differential equation, stability analysis, Lyapunov function, zero-Hopf bifurcation

\section{INTRODUCTION}

Human immunodeficiency virus (HIV) is a retrovirus that attacks and destroys the immune system, so that it causes the HIV infection [10] and the disease called AIDS (Acquired Immune Deficiency Syndrome [8, 24,30]). AIDS is a condition in humans in which the progressive collapse of the immune system allows infections and cancers which threat human life. According to data published by the World Health Organization (WHO), it is presumed that more than 35 million people died due to AIDS since it was first discovered in 1981, and 37.9 million people were living with HIV at the end of 2018. There is no cure for AIDS but there are certain medicines that are used to slow down this disease. 
The main target of HIV is the immune system, especially the CD4 ${ }^{+}$T-cells, which are the most sufficient white blood cells, or lymphocytes. $\mathrm{CD}^{+} \mathrm{T}$ lymphocytes play a central role in orchestrating the beginning and maintenance of the adaptive immune response $[2,13]$. These cells are more commonly referred to as helper T-cells which is the term we will use in this paper. The helper T-cells can be categorized as effector and non-effector helper T-cells. The effector helper T-cells are triggered by the tumour cell proliferation, and they have a death rate which is considerably small compare to the normal helper T-cells. These cells are mediated by the cytokines secreted by the differentiated cells. Therefore, they are specific to certain disease [2]. HIV causes the destruction of helper T-cells; as a result, it decreases the body's ability to fight the infection. The count of helper T-cell is normally around $1000 \mathrm{~mm}^{-3}$ in a human being. However, when it decreases to $200 \mathrm{~mm}^{-3}$ or below in an HIV infected individual, then that person is classified as having AIDS [15,26].

HIV infected individuals have a high risk of developing certain tumours. The connection between HIV/AIDS and certain tumours has not been understood completely, but it likely depends on a weakened immune system $[4,6,33,36]$. When normal cells begin to change and grow uncontrollably, a mass called tumour forms. A tumour can be benign, also called non-cancerous, or malignant which is cancerous (see the reference [1] for more details). Malignancies that are found to have a high incidence of HIV-infected individuals are Kaposi's sarcoma (KS), Hodgkin's disease (HD), non-Hodgkin's lymphoma (NHL), squamous cell carcinomas, plasmacytomas and leiomyosarcoma in children [36]. In the tumour cells of HIV infected patients, no viral sequence in the DNA was found; therefore, it seems that the virus doesn't include the tumour itself [20]. Furthermore, the immune surveillance hypothesis explains that the immune system patrols the body to recognize and destroy invading pathogens $[6,7]$.

There is a variety of mathematical models that studied and analyzed the tumour growth [19,27]. Also, in recent years, modeling, analysis and control of the HIV infection have attracted the attention of researchers in bio-mathematics (see, for example, $[11,25,26]$ and references cited therein). However, the interaction between both the HIV-immune system and the tumour-immune system has not been fully understood, since several types of tumours are associated to the HIV occurrence. Therefore, it is important to investigate the dynamics of the immune system in this situation. A few of such studies can be found in [5, 12, 21, 22, 29].

The aim of this work is to better understand the interaction between tumour and immune system in an HIV infected individual. More precisely, we want to analyze how the existence of HIV infection affects the dynamics of the immune system and tumour cells. To do this, we use a system of delay differential equations where the time lag describes the time needed by helper T-cells to find (or it can be said 'recognize') tumour cells and virus. Inspired by the studies in [12,29], we present a mathematical model involving four populations: tumour cells, uninfected effector 
helper T-cells, infected helper T-cells and free virus. The models presented in this manuscript and also [12,29] are improved from the model introduced in the papers by Lou et al. [21, 22]. In [22], Lou et al. presented a model of cell-to-cell spread of HIV together with tumour in tissue cultures (in vitro). They aimed at explaining some properties concerning tumour occurring during the HIV infection. The same model is studied with the delay in [21].

In [5], Bodnar et al. proposed a model to describe the HIV related tumour-immune system interactions in vitro. Moreover, in [12], Forys and Poleszczuk considered a similar model. However, they considered the issue of immune reaction against tumour and the second way for HIV to disseminate in vivo (circulating free viral particles to T-cells directly). In [29], Rihan and Rahman studied a model which describes the interaction between tumour cells, the general population of the helper T-cells, infected helper T-cells and virus where the time lag is considered to represent the time needed by the healthy effector cells to recognize the tumour cells and virus. Also, they consider that HIV disseminates in vivo by circulating free viral particles to T-cells directly.

The difference between the study in [29] and the present paper is that the model we examined here involves two different discrete delays, and its stability and bifurcation analysis is given for the full model, i.e., without reducing the dimension of the model proposed. In addition, the difference between the study in [12] and this work is that the helper T-cells that we consider are exactly the effector helper T-cells, not general ones. HIV attacks the helper T-cells but then each helper T-cell is target to the virus. Namely, we care more about the cells that are specific to tumour and what happened to them is more important for us.

In this paper, the existence, uniqueness and non-negativity of solutions, and also both local and global stabilities of steady states of the model without delay are first studied. And then, the existence of a zero-Hopf bifurcation for the model with delay is given. Apart from Section 1, the paper is organized in the following aspect: In Section 2, we introduce a mathematical model of HIV infection with tumour cells; the mathematical analysis of the ODE model is performed. The theoretical results about steady states and their stabilities are presented in Section 3. Later, the analysis is presented for the DDE model in Section 4. The existence of a zero-Hopf bifurcation is investigated in this section. Numerical simulations that support and extend the theoretical results are given in Section 5. Section 6 is devoted to conclusions and predictions of the models.

\section{THE MODEL}

We consider the following delay differential equation system describing the tumourimmune system interactions in the case of HIV: 


$$
\begin{aligned}
\frac{d T}{d t} & =r_{1} T(t)-k_{1} T(t) E\left(t-\tau_{1}\right), \\
\frac{d E}{d t} & =r_{2} T(t)-\mu_{1} E(t)-\theta k_{1} T(t) E\left(t-\tau_{1}\right)-k_{2} E\left(t-\tau_{2}\right) V(t), \\
\frac{d I}{d t} & =k_{2} E\left(t-\tau_{2}\right) V(t)-c I(t), \\
\frac{d V}{d t} & =N \delta I(t)-\mu_{2} V(t),
\end{aligned}
$$

where $T(t), E(t), I(t), V(t)$ denote the concentration of tumour cells, healthy effector helper T-cells, helper T-cells infected by free HIVs and free HIV particles at time $t$, respectively, and all parameters are positive. The time lags $\tau_{1}$ and $\tau_{2}$ describe the time needed by the helper T-cells to recognize tumour cells and virus, respectively.

We assume that the tumour cells grow exponentially with a constant proliferation rate; we do not consider resource limitation. Such type of tumour growth is experimentally observed at the beginning of the tumour development [35]. Also, we assume the linear response of the immune system to tumour cell presence. In the model, we take this response as proportional to the multiplication of both tumour and immune system cell concentrations.

Healthy effector helper T-cells are reproduced as a result of the presence of tumour. The parameter $r_{2}$ indicates the antigenicity of tumour. Antigenicity can be thought of as a measure of how different the tumour cells are from normal cells [16]. Parameters $\mu_{1}, c$ and $\mu_{2}$ are natural death rates of the healthy T-cells, the infected T-cells and the HIV particles, respectively, because cells have a finite life span. Also, the parameter $\theta$ represents the small percentage of T-cells that do not survive after killing the tumour cells (the inequality $\theta \leq 1$ is obvious because of the definition of this parameter).

HIV can spread out in vivo either by transmission of cell-free virus or directly from cell-to-cell via the formation of virological synapses as stated in $[9,17,18]$. In this model, similar to [11], we assume that the transition of the healthy T-cells into the infected ones is due to direct interaction with the virus. Accordingly, the infection rate is given by $k_{2}$ which increases the count of the infected helper T-cells.

Finally, according to [26], the virus is produced by the productively infected Tcells. Here, we have assumed that on average each productively infected helper Tcell produces $\mathrm{N}$ virus during its lifetime. Since the average lifetime of a productively infected cell is $\frac{1}{\delta}$, the average rate of virus production is $N \delta$. Therefore, $N \delta$ represents the source for free viruses. In this derivation, we have ignored the loss of virus due to the infection of a cell. 


\section{ANALYSIS OF THE ODE MODEL}

In this section, we study the model without delay. By taking $\tau_{1}=\tau_{2}=0$ in system (2.1) we obtain the following ODE model:

$$
\begin{aligned}
\frac{d T}{d t} & =r_{1} T(t)-k_{1} T(t) E(t), \\
\frac{d E}{d t} & =r_{2} T(t)-\mu_{1} E(t)-\theta k_{1} T(t) E(t)-k_{2} E(t) V(t), \\
\frac{d I}{d t} & =k_{2} E(t) V(t)-c I(t), \\
\frac{d V}{d t} & =N \delta I(t)-\mu_{2} V(t),
\end{aligned}
$$

where all parameters and variables are the same as described in the former section.

One can write system (3.1) as a vector equation form as follows:

$$
\frac{d X(t)}{d t}=F(t, X(t))
$$

where $X(t)=(T(t), E(t), I(t), V(t))^{T}$ and $F(t)=\left(f_{1}(t), f_{2}(t), f_{3}(t), f_{4}(t)\right)^{T}$ in which

$$
\begin{aligned}
& f_{1}(t)=r_{1} T(t)-k_{1} T(t) E(t), \\
& f_{2}(t)=r_{2} T(t)-\mu_{1} E(t)-\theta k_{1} T(t) E(t)-k_{2} E(t) V(t), \\
& f_{3}(t)=k_{2} E(t) V(t)-c I(t), \\
& f_{4}(t)=N \delta I(t)-\mu_{2} V(t) .
\end{aligned}
$$

\subsection{Positivity of solutions}

The following Lemma underlines that for positive initial data, the solution of system (3.1) uniquely exists and remains in $\mathbb{R}_{+}^{4}$. From biological point of view, it means that the model is reasonable in the sense that no population becomes negative. Therefore, there is no need analyzing of the trivial steady state of system (3.1).

Lemma 1. The solution of system (3.1) with non-negative initial conditions $T_{0}$, $E_{0}, I_{0}$ and $V_{0}$ uniquely exists and remains in $\mathbb{R}_{+}^{4}$.

Proof. Note that $F(t, X(t))$ in Eq. (3.2) is continuous and also Lipschitz with respect to $X(t)$ on any four-dimensional box $D$. Then system (3.1) has the unique solution on $[0, b)$ where $b$ can be determined as $t \rightarrow b^{-}$at which either the solution becomes unbounded or the solution approaches to the boundary of $D$. In addition, We assume that $T(t), E(t), I(t)$ and $V(t)$ initially have positive values. Recall that all constants in the system are positive. For positive initial conditions $T_{0}, E_{0}, I_{0}$ and $V_{0}$, from the first and the second equations of system (3.1) we have the following (where $A(t)$ is the integrating factor):

$$
T(t)=T_{0} e^{\int_{0}^{t} r_{1}-k_{1} E(s) d s} \geq 0, \quad \forall t \geq 0 .
$$




$$
E(t)=\frac{A(0) E_{0}+r_{2} \int_{0}^{t} A(s) T(s) d s}{A(t)} \geq 0, \quad \forall t \geq 0 .
$$

From the third and the fourth equations of the system, we have

$$
\begin{gathered}
I(t)=I_{0} e^{-c t}+k_{2} e^{-c t} \int_{0}^{t} e^{c s} E(s) V(s) d s, \\
V(t)=V_{0} e^{-\mu_{2} t}+N \delta e^{-\mu_{2} t} \int_{0}^{t} e^{\mu_{2} s} I(s) d s .
\end{gathered}
$$

Let us denote by $t_{*}$ the first time for which one of the populations $I(t)$ and $V(t)$ become zero, or more precisely $\min \left\{I\left(t_{*}\right), V\left(t_{*}\right)\right\}=0$. Without loss of generality, let $V\left(t_{*}\right)=0$. So, $I\left(t_{*}\right)>0$ for $t \in\left[0, t_{*}\right]$ since $t_{*}$ is the first time for which one of the populations $I(t)$ and $V(t)$ become zero. Also $V(t)>0$ for $t \in\left[0, t_{*}\right)$ since we assume that $T(t), E(t), I(t)$ and $V(t)$ initially have non-negative values. Therefore, $V(t)$ must be non-increasing on $\left[0, t_{*}\right]$, or more precisely

$$
\left.\frac{d V}{d t}\right|_{t=t_{*}} \leqslant 0
$$

On the other hand, one can see that from the last equation of system (3.1)

$$
\left.\frac{d V}{d t}\right|_{t=t_{*}}=N \delta I\left(t_{*}\right)-\mu_{2} V\left(t_{*}\right)=N \delta I\left(t_{*}\right)>0
$$

since the equation $V\left(t_{*}\right)=0$ holds. Consequently, this leads to a contradiction. Thus, there cannot be found a $t_{*}$ such that $V\left(t_{*}\right)=0$. So, for $\forall t>0, V(t)>0$ and $I(t)>0$. This completes the proof.

\subsection{Steady states}

In order to fully understand the dynamics of the model, first we must establish the values of steady states. The steady states of system (3.1) can be obtained by setting the equations $f_{1}(t), f_{2}(t), f_{3}(t), f_{4}(t)$ simultaneously equal to zero. The following lemma explains the steady states of the model.

Lemma 2. Let

$$
\Re_{1}=r_{2}-\theta r_{1} \text { and } \Re_{0}=\sqrt{\frac{N \delta k_{2} r_{1}}{c k_{1} \mu_{2}}} .
$$

If $\Re_{1}>0$, then system (3.1) has two non-negative steady states other than the trivial one:

(1) If $\Re_{0} \neq 1$, then one obtains the non-infected steady state $S_{0}=\left(\frac{\mu_{1} r_{1}}{k_{1}\left(r_{2}-\theta r_{1}\right)}, \frac{r_{1}}{k_{1}}, 0,0\right)$.

(2) If $\Re_{0}=1$, then one obtains the steady state $S^{*}=\left(\frac{\mu_{1} r_{1}+k_{2} r_{1} \vartheta}{k_{1}\left(r_{2}-\theta r_{1}\right)}, \frac{r_{1}}{k_{1}}, \frac{r_{1} k_{2}}{k_{1} c} \vartheta, \vartheta\right)$, where $\vartheta \in \mathbb{R}^{+} \cup\{0\}$. 


\subsection{Local stability analysis}

The fact that the stability properties depend on the eigenvalues of the system is well-known for linear ODEs. However, our model is nonlinear, and thus we must use linearization. We will investigate the local stability properties of the steady states by approximating the nonlinear system of differential equations with a linear system at the points $S_{0}$ and $S^{*}$. The local stability analysis of these steady states is given below.

Theorem 1. For system (3.1), if $\Re_{1}>0$ and $\Re_{0}<1$, then the steady state $S_{0}$ is locally asymptotically stable. Furthermore, the steady state $S^{*}$ is always L-stable.

Proof. First, we linearize system (3.1) around its steady states and then find its Jacobian matrices as follows:

$$
J_{S_{0}}=\left[\begin{array}{cccc}
0 & -\frac{\mu_{1} r_{1}}{r_{2}-\theta \theta_{1}} & 0 & 0 \\
r_{2}-\theta r_{1} & -\mu_{1}-\frac{\theta \mu_{1} r_{1}}{r_{2}-\theta r_{1}} & 0 & -\frac{r_{1} k_{2}}{k_{1}} \\
0 & 0 & -c & \frac{r_{1} k_{2}}{k_{1}} \\
0 & 0 & N \delta & -\mu_{2}
\end{array}\right]
$$

and

$$
J_{S^{*}}=\left[\begin{array}{cccc}
0 & -r_{1} \frac{\mu_{1}+k_{2} \vartheta}{r_{2}-\theta r_{1}} & 0 & 0 \\
r_{2}-\theta r_{1} & -\mu_{1}-k_{2} \vartheta-\theta r_{1}\left(\frac{\mu_{1}+k_{2} \vartheta}{r_{2}-\theta r_{1}}\right) & 0 & -\frac{r_{1} k_{2}}{k_{1}} \\
0 & k_{2} \vartheta & -c & \frac{r_{1} k_{2}}{k_{1}} \\
0 & 0 & N \delta & -\mu_{2}
\end{array}\right] \text {. }
$$

If all eigenvalues of the Jacobian matrix have negative real parts, then the steady state is locally asymptotically stable. Characteristic equation of $J_{S_{0}}$ is given by

$$
P(\lambda)=\lambda^{4}+a_{1} \lambda^{3}+a_{2} \lambda^{2}+a_{3} \lambda+a_{4},
$$

where

$a_{1}=\frac{1}{r_{2}-\theta r_{1}}\left(\left(c+\mu_{2}\right)\left(r_{2}-\theta r_{1}\right)+\mu_{1} r_{2}\right)$,

$a_{2}=\frac{1}{k_{1}\left(r_{2}-\theta r_{1}\right)}\left(\left(c \mu_{2} k_{1}-N \delta k_{2} r_{1}\right)\left(r_{2}-\theta r_{1}\right)+\mu_{1} k_{1} r_{2}\left(c+\mu_{2}\right)+\mu_{1} k_{1} r_{1}\left(r_{2}-\theta r_{1}\right)\right)$,

$a_{3}=\frac{\mu_{1}}{k_{1}\left(r_{2}-\theta r_{1}\right)}\left(k_{1} r_{1}\left(c+\mu_{2}\right)\left(r_{2}-\theta r_{1}\right)+r_{2}\left(c \mu_{2} k_{1}-N \delta k_{2} r_{1}\right)\right)$,

$a_{4}=\frac{\mu_{1} r_{1}}{k_{1}}\left(c \mu_{2} k_{1}-N \delta k_{2} r_{1}\right)$.

Also, one can calculate that 


$$
\begin{aligned}
a_{1} a_{2} a_{3}-a_{3}^{2}-a_{1}^{2} a_{4}= & \frac{\mu_{1} r_{2}\left(c+\mu_{2}\right)}{k_{1}^{2}\left(r_{2}-\theta r_{1}\right)^{3}} . \\
& \left(\begin{array}{c}
\left(r_{2}-\theta r_{1}\right)^{2}\left(c \mu_{2} k_{1}-N \delta k_{2} r_{1}-\mu_{1} k_{1} r_{1}\right)^{2} \\
+\left(c+\mu_{2}\right)\left(r_{2}-\theta r_{1}\right)\left(c \mu_{2} k_{1}-N \delta k_{2} r_{1}\right) \mu_{1} k_{1} r_{2} \\
+\left(c+\mu_{2}\right)\left(r_{2}-\theta r_{1}\right) \mu_{1}^{2} k_{1}^{2} r_{1} r_{2}+\left(c \mu_{2} k_{1}-N \delta k_{2} r_{1}\right) \mu_{1}^{2} k_{1} r_{2}^{2} \\
+\left(c+\mu_{2}\right)^{2}\left(r_{2}-\theta r_{1}\right)^{2} \mu_{1} k_{1}^{2} r_{1}
\end{array}\right) .
\end{aligned}
$$

Here, we know all parameters are positive. If $\Re_{0}<1$, then we obtain $a_{1} a_{2} a_{3}-a_{3}^{2}-$ $a_{1}^{2} a_{4}>0$. So, because of the Routh-Hurwitz criteria all eigenvalues of the Jacobian matrix for $S_{0}$ have negative real parts. Thus, the steady state $S_{0}$ is locally asymptotically stable.

However, for the steady state $S^{*}$ the characteristic equation has the form of

$$
P(\lambda)=\lambda^{4}+a_{1} \lambda^{3}+a_{2} \lambda^{2}+a_{3} \lambda+a_{4},
$$

where

$$
\begin{aligned}
& a_{1}=c+\mu_{1}+\mu_{2}+k_{2} \vartheta+\theta r_{1} \frac{\mu_{1}+k_{2} \vartheta}{r_{2}-\theta r_{1}}, \\
& a_{2}=\theta r_{1}\left(c+\mu_{2}\right) \frac{\mu_{1}+\vartheta k_{2}}{r_{2}-\theta r_{1}}+\left(\vartheta k_{2}+\mu_{1}\right)\left(c+\mu_{2}+r_{1}\right), \\
& a_{3}=c \mu_{1} r_{1}+\mu_{1} \mu_{2} r_{1}+c \vartheta \mu_{2} k_{2}+c \vartheta k_{2} r_{1}+\vartheta \mu_{2} k_{2} r_{1}, \\
& a_{4}=0 .
\end{aligned}
$$

Here, one of the eigenvalues is equal to zero. Applying the Routh-Hurwitz criteria to the reduced characteristic equation below:

$$
P(\lambda)=\lambda^{3}+a_{1} \lambda^{2}+a_{2} \lambda+a_{3}+a_{4}
$$

we get three remaining eigenvalues of the Jacobian matrix that have negative real parts. Therefore, the steady state $S^{*}$ is Lyapunov stable (L-stable) (see the reference [3] for the definition of Lyapunov Stability).

\subsection{Global stability analysis}

In this section, our aim is to investigate the long time behavior of the given system by analyzing global stability. Let's start this section with a remark. Next, we prove the global stability of the disease-free steady state.

Remark 1 . The steady state $S^{*}$ cannot be globally stable since it is L-stable. Some numerical simulations that support this observation will be given later.

Theorem 2. For system (3.1), if $\Re_{1}>0$ and $\Re_{0}<1$, then the disease free steady state $S_{0}$ is globally asymptotically stable on $\Gamma$ where

$$
\Gamma=\left\{(T, E, I, V) \in \mathbb{R}_{+}^{4}: T+E+I+V \leq \frac{r_{1}}{k_{1}}\right\} .
$$


Proof. First, system (3.1) can be thought as a compartmental model, i.e., the system can be written as follows:

$$
\begin{aligned}
& \frac{d x}{d t}=\mathcal{F}(x, y)-\mathcal{V}(x, y) \\
& \frac{d y}{d t}=g(x, y)
\end{aligned}
$$

with $g=\left(g_{1}, g_{2}\right)^{T}$ where $g_{1}=r_{1} T-k_{1} T E$ and $g_{2}=r_{2} T-\mu_{1} E-\theta k_{1} T E-k_{2} E V$. Here, $x=(I, V)^{T}$ and $y=(T, E)^{T}$ represent the populations in disease compartments and non-disease compartments, respectively. In addition, $\mathcal{F}=\left(\mathcal{F}_{1}, \mathcal{F}_{2}\right)^{T}$ and $\mathcal{V}=$ $\left(\mathcal{V}_{1}, \mathcal{V}_{2}\right)^{T}$ where $\mathcal{F}_{1}=k_{2} E V, \mathcal{F}_{2}=N \delta I$ represent the rates of new infections in the disease compartments, and $\mathcal{V}_{1}=c I, \mathcal{V}_{2}=\mu_{2} V$ represent the transition terms in the disease compartments.

One can easily check that following assumptions are satisfied in order to ensure the model (3.5) is well posed:

(1) $\mathcal{F}_{i}(0, y)=0$ and $\mathcal{V}_{i}(0, y)=0$ for all $y \geq 0$ and $i=1,2$.

(2) $\mathcal{F}_{i}(x, y) \geq 0$ for all non-negative $x$ and $y$ and $i=1,2$.

(3) $\mathcal{V}_{i}(x, y) \leq 0$ whenever $x_{i}=0, i=1,2$.

(4) $\mathcal{V}_{1}(x, y)+\mathcal{V}_{2}(x, y) \geq 0$ for all non-negative $x$ and $y$.

(5) The disease free system $\frac{d y}{d t}=g(0, y)$ has a unique equilibrium that is locally asymptotically stable. This equilibrium point is $y_{0}=\left(T_{0}, E_{0}\right)=\left(\frac{\mu_{1} r_{1}}{k_{1}\left(r_{2}-\Theta r_{1}\right)}, \frac{r_{1}}{k_{1}}\right)$.

Therefore, the linearized equations for the disease compartments can be written as follows:

$$
\frac{d x}{d t}=(F-V) x,
$$

where $F$ and $V$ are $2 \times 2$ matrices with entries

$$
F=\frac{\partial \mathcal{F}_{i}}{\partial x_{j}}\left(0, y_{0}\right)=\left[\begin{array}{cc}
0 & \frac{k_{2} r_{1}}{k_{1}} \\
N \delta & 0
\end{array}\right] \text { and } V=\frac{\partial \mathcal{V}_{i}}{\partial x_{j}}\left(0, y_{0}\right)=\left[\begin{array}{cc}
c & 0 \\
0 & \mu_{2}
\end{array}\right]
$$

Hence, the next generation matrix is

$$
K=F V^{-1}=\left[\begin{array}{cc}
0 & \frac{k_{2} r_{1}}{k_{1}} \\
N \delta & 0
\end{array}\right]\left[\begin{array}{cc}
\frac{1}{c} & 0 \\
0 & \frac{1}{\mu_{2}}
\end{array}\right]=\left[\begin{array}{cc}
0 & \frac{k_{2} r_{1}}{\mu_{2} k_{1}} \\
\frac{N \delta}{c} & 0
\end{array}\right]=\left[\begin{array}{cc}
0 & \frac{k_{2} E_{0}}{\mu_{2}} \\
\frac{N \delta}{c} & 0
\end{array}\right],
$$

and the reproduction number $\mathcal{R}_{0}$ can be defined as the spectral radius of $K$ (see the references [14], [31], [34]), that can be calculated as

$$
\mathcal{R}_{0}=\sqrt{\frac{N \delta k_{2} r_{1}}{c \mu_{2} k_{1}}}=\sqrt{\frac{N \delta k_{2} E_{0}}{c \mu_{2}}} .
$$


One can easily show that the biologically feasible region

$$
\Gamma=\left\{(T, E, I, V) \in \mathbb{R}^{4}: T+E+I+V \leq \frac{r_{1}}{k_{1}}\right\}
$$

is positively invariant. On the other hand, set

$$
f(x, y)=\left[\begin{array}{c}
k_{2} V\left(E_{0}-E\right) \\
0
\end{array}\right] .
$$

Then for the disease compartments can be written as

$$
\frac{d x}{d t}=(F-V) x-f(x, y) .
$$

So, the Lyapunov function on $\Gamma$ can be constructed as in [14] and [31]. $Q=W^{T} V^{-1} x$ is a Lyapunov function where $W^{T}$ be the left eigenvector of $V^{-1} F$. A straightforward calculation gives

$$
Q=W^{T} V^{-1} x=\left[\begin{array}{ll}
1 & \frac{k_{2} r_{1} \sqrt{c \mu_{2} k_{1}}}{k_{1} c \sqrt{N \delta k_{2} r_{1}}}
\end{array}\right]\left[\begin{array}{cc}
\frac{1}{c} & 0 \\
0 & \frac{1}{\mu_{2}}
\end{array}\right]\left[\begin{array}{c}
I \\
V
\end{array}\right]=\frac{1}{c} I+\frac{k_{2} r_{1} \sqrt{c \mu_{2} k_{1}}}{k_{1} c \mu_{2} \sqrt{N \delta k_{2} r_{1}}} V .
$$

One can now easily verify that $Q$ is a Lyapunov function on $\Gamma$ provided $\mathcal{R}_{0}<1$ as follows:

$$
\begin{aligned}
\frac{d Q}{d t} & =\frac{1}{c}\left(k_{2} E V-c I\right)+\frac{k_{2} r_{1} \sqrt{c \mu_{2}}}{k_{1} c \mu_{2} \sqrt{N \delta k_{2} E_{0}}}\left(N \delta I-\mu_{2} V\right) \\
& =V\left(\frac{k_{2} E}{c}-\frac{k_{2} r_{1} \sqrt{c \mu_{2}}}{k_{1} c \sqrt{N \delta k_{2} E_{0}}}\right)+I\left(\frac{N \delta k_{2} r_{1} \sqrt{c \mu_{2}}}{k_{1} c \mu_{2} \sqrt{N \delta k_{2} E_{0}}}-1\right) \\
& =V\left(\frac{k_{2} E}{c}-\frac{k_{2} r_{1} \sqrt{c \mu_{2}}}{k_{1} c \sqrt{N \delta k_{2} E_{0}}}\right)+I\left(\frac{\sqrt{N \delta k_{2} r_{1}}}{\sqrt{k_{1} c \mu_{2}}}-1\right)<0 .
\end{aligned}
$$

Hence $Q$ is a Lyapunov function on $\Gamma$, and the largest compact invariant set in $\left\{(T, E, I, V) \in \Gamma: \frac{d Q}{d t}=0\right\}$ is $\left\{S_{0}\right\}$. Thus, by LaSalle's invariance principle, every solution of system (3.1) with initial conditions in $\Gamma$ approaches $S_{0}$ as $t \rightarrow \infty$ whenever $\mathcal{R}_{0}<1$. Therefore, the disease-free equilibrium point $S_{0}$ is globally asymptotically stable on $\Gamma$ for $\mathcal{R}_{0}<1$.

\section{ANALYSis OF THE DDE MODEL}

In this section, we study the local stability of the equilibria and the existence of zero-Hopf bifurcation in system (2.1) by dividing it into the following cases due to the delay:

(1) $\tau_{1}=\tau_{2}=\tau$,

(2) $\tau_{1}>0$ and $\tau_{2}=0$,

(3) $\tau_{1}>0$, and $\tau_{2}>0$ but $\tau_{1} \neq \tau_{2}$. 
Note that the case without delay $\left(\tau_{1}=\tau_{2}=0\right)$ is already analyzed in the former section. Also, note that the steady states of system (2.1) is the same as those of system (3.1). Therefore, Lemma 2 still holds for system (2.1). We now want to analyze the system for the endemic equilibrium point $S^{*}$ which exists under the condition $\mathfrak{R}_{0}=1$.

\subsection{Bifurcation analysis of system (2.1) when $\tau_{1}=\tau_{2}=\tau$}

Under the condition $\Re_{0}=1$, the corresponding linearized system at $S^{*}$ as follows:

$$
\begin{aligned}
\frac{d T}{d t} & =a_{13} E(t-\tau), \\
\frac{d E}{d t} & =a_{21} T(t)+a_{22} E(t)+a_{23} E(t-\tau)+a_{25} V(t), \\
\frac{d I}{d t} & =a_{33} E(t-\tau)+a_{34} I(t)+a_{35} V(t), \\
\frac{d V}{d t} & =a_{44} I(t)+a_{45} V(t),
\end{aligned}
$$

where

$$
\begin{array}{ll}
a_{13}=-\frac{r_{1}\left(\mu_{1}+k_{1} \vartheta\right)}{r_{2}-\theta r_{1}}, & a_{21}=r_{2}-\theta r_{1}, \\
a_{22}=-\mu_{1}, & a_{23}=-\frac{r_{2} k_{2} \vartheta-\theta r_{1} \mu_{1}}{r_{2}-\theta r_{1}}, \\
a_{25}=-\frac{k_{2} r_{1}}{k_{1}}, & a_{33}=k_{2} \vartheta, \\
a_{34}=-c, & a_{35}=\frac{k_{2} r_{1}}{k_{1}} \\
a_{43}=N \delta, & a_{45}=-\mu_{2} .
\end{array}
$$

Therefore, for this case corresponding characteristic equation of system (4.1) becomes:

$$
P(\lambda, \tau) \equiv \lambda^{4}+a_{1} \lambda^{3}+a_{2} \lambda^{2}+e^{-\lambda \tau}\left(b_{1} \lambda^{3}+b_{2} \lambda^{2}+b_{3} \lambda\right)=0,
$$

where

$$
\begin{aligned}
& a_{1}=c+\mu_{1}+\mu_{2}, \\
& a_{2}=\mu_{1}\left(c+\mu_{2}\right), \\
& b_{1}=\frac{k_{2} \vartheta r_{2}+\mu_{1} \theta r_{1}}{r_{2}-\theta r_{1}}, \\
& b_{2}=\left(\frac{k_{2} \vartheta r_{2}+\mu_{1} \theta r_{1}}{r_{2}-\theta r_{1}}\right)\left(c+\mu_{2}\right)+r_{1}\left(\mu_{1}+k_{2} \vartheta\right), \\
& b_{3}=r_{1}\left(\mu_{1} k_{2} \vartheta\right)\left(c+\mu_{2}\right)+c \mu_{2} k_{2} \vartheta .
\end{aligned}
$$


$P(\lambda, \tau)$ in Eq. (4.2) is a transcendental polynomial and has infinitely many roots. First, it is easy to see that one of its roots is zero. This is a simple root since $a_{2}$ and $b_{3}$ are positive because of the positivity of the parameters of the model. Notice that when $\tau=0$ in Eq. (4.2), one obtains the characteristic equation of the ODE system in which the distribution of its eigenvalues is already studied in the former section.

In this section, our aim is to investigate the existence of the zero-Hopf bifurcation. In other words, we will determine the conditions on the parameters for the occurrence of a zero-Hopf bifurcation. To do this, we first need to show the existence of a pair of purely imaginary roots for Eq. (4.2).

Let us denote $\lambda=\eta(\tau)+i w(\tau)$. We look for a pair of purely imaginary roots such that $\lambda\left(\tau^{*}\right)=i \omega\left(\tau^{*}\right)=i \omega_{0}, \omega_{0}>0$ (without loss of generality). Notice that if such an $\omega\left(\tau^{*}\right)=\omega_{0}$ does not exist, then the steady state $S^{*}$ stays stable forever because of the continuity. It is clear that $\lambda=i \omega, \omega>0$, is a root of Eq. (4.2) if

$$
(i \omega)^{3}+a_{1}(i \omega)^{2}+a_{2}(i \omega)+e^{-(i \omega) \tau}\left(b_{1}(i \omega)^{3}+b_{2}(i \omega)+b_{3}\right)=0 .
$$

Separating the real and imaginary parts, we obtain the following equations:

$$
\begin{array}{ll}
-a_{1} \omega^{2}-b_{1} \cos (\omega \tau) \omega^{2}+b_{3} \cos (\omega \tau)+b_{2} \sin (\omega \tau) \omega & =0, \\
-\omega^{3}+a_{2} \omega+b_{2} \cos (\omega \tau) \omega+b_{1} \sin (\omega \tau) \omega^{2}-b_{3} \sin (\omega \tau) & =0 .
\end{array}
$$

Squaring first both sides of these equations and then adding them up, one reaches to the following equation:

$$
\omega^{6}+\left(a_{1}^{2}-2 a_{2}-b_{1}^{2}\right) \omega^{4}+\left(a_{2}^{2}+2 b_{1} b_{3}-b_{2}^{2}\right) \omega^{2}-b_{3}^{2}=0 .
$$

Now, let us take $\omega^{2}=z$, then we can rewrite Eq. (4.4) as follows:

$$
z^{3}+\left(a_{1}^{2}-2 a_{2}-b_{1}{ }^{2}\right) z^{2}+\left(a_{2}^{2}+2 b_{1} b_{3}-b_{2}{ }^{2}\right) z-b_{3}{ }^{2}=0 .
$$

It is obvious that $-b_{3}{ }^{2}<0$. Also, by the Vieta's Theorem [23], it is known that the expression $b_{3}{ }^{2}$ is equal to the product of the roots. Therefore, the product of the roots of Eq. (4.5) is positive. Then, it is clear that at least one of them is positive since Eq. (4.5) has at most three roots. As a result of this, it is assured that there is at least one positive root $\omega_{0}$ of Eq. (4.4), too. Thus, the characteristic equation (4.2) has a pair of purely imaginary roots $\pm i \omega_{0}$ at $\tau^{*}$. Now, if we solve $\sin (\omega \tau)$ and $\cos (\omega \tau)$ from Eq. (4.3) simultaneously, we obtain the following equations:

$$
\begin{aligned}
& \sin (\omega \tau)=\frac{b_{2} a_{1} \omega^{3}-\left(b_{3}-b_{1} \omega^{2}\right)\left(\omega^{3}-a_{2} \omega\right)}{b_{2}^{2} \omega^{2}+\left(b_{1} \omega^{2}-b_{3}\right)^{2}}, \\
& \cos (\omega \tau)=\frac{a_{1} \omega^{2}\left(b_{3}-b_{1}^{2}\right)+b_{2} \omega^{2}\left(\omega^{2}-a_{2}\right)}{b_{2}^{2} \omega^{2}+\left(b_{1} \omega^{2}-b_{3}\right)^{2}} .
\end{aligned}
$$

Finally, utilizing these equations we solve $\tau_{n}$ for $n=0,1,2, \ldots$ as follows:

$$
\tau_{n}=\frac{1}{\omega_{0}} \arccos \left(\frac{a_{1} \omega_{0}^{2}\left(b_{3}-b_{1} \omega_{0}^{2}\right)+b_{2} \omega_{0}^{2}\left(\omega_{0}^{2}-a_{2}\right)}{b_{2}^{2} \omega_{0}^{2}+\left(b_{1} \omega_{0}^{2}-b_{3}\right)^{2}}\right)+\frac{2 \pi n}{\omega_{0}} .
$$


Let us denote $\tau^{*}=\min \left\{\tau_{n}: n=0,1,2, ..\right\}>0$ such that $i \omega_{0}=i \omega\left(\tau^{*}\right)$ is a root of Eq. (4.2). Now, differentiating both sides of Eq. (4.2) with respect to $\tau$, and then utilizing Eq. (4.2), we derive the following equation:

$$
\begin{aligned}
\left(\frac{d \lambda}{d \tau}\right)^{-1} & =-\frac{\left(3 \lambda^{2}+2 a_{1} \lambda+a_{2}\right)-\tau e^{-\lambda \tau}\left(b_{1} \lambda^{2}+b_{2} \lambda+b_{3}\right)+e^{-\lambda \tau}\left(2 b_{1} \lambda+b_{2}\right)}{-\lambda e^{-\lambda \tau}\left(b_{1} \lambda^{2}+b_{2} \lambda+b_{3}\right)} \\
& =\frac{\left(3 \lambda^{2}+2 a_{1} \lambda+a_{2}\right) e^{\lambda \tau}}{\lambda\left(b_{1} \lambda^{2}+b_{2} \lambda+b_{3}\right)}-\frac{2 b_{1} \lambda+b_{2}}{\lambda\left(b_{1} \lambda^{2}+b_{2} \lambda+b_{3}\right)}-\frac{\tau}{\lambda} \\
& =-\frac{3 \lambda^{2}+2 a_{1} \lambda+a_{2}}{\lambda^{4}+a_{1} \lambda^{3}+a_{2} \lambda^{2}}-\frac{2 b_{1} \lambda+b_{2}}{b_{1} \lambda^{3}+b_{2} \lambda^{2}+b_{3} \lambda}-\frac{\tau}{\lambda} .
\end{aligned}
$$

Thus, its reel part has the form of

$$
\left.\operatorname{Re}\left(\frac{d \lambda}{d \tau}\right)^{-1}\right|_{\tau=\tau^{*}}=\frac{\omega_{0}^{2}\left(3 \omega_{0}^{4}+\left(2 a_{1}^{2}-4 a_{2}\right) \omega_{0}^{2}+a_{2}^{2}\right)}{\left(\omega_{0}^{4}-a_{2} \omega_{0}^{2}\right)^{2}+\left(a_{1} \omega_{0}^{3}\right)^{2}}-\frac{-2 b_{1}^{2} \omega_{0}^{4}+\left(2 b_{1} b_{3}-b_{2}^{2}\right) \omega_{0}^{2}}{\left(b_{2} \omega_{0}^{2}\right)^{2}+\left(b_{3} \omega_{0}-b_{1} \omega_{0}^{3}\right)^{2}}>0
$$

since $2 a_{1}^{2}-4 a_{2}>0$ and $2 b_{1} b_{3}-b_{2}^{2}<0$. Hence, the transversality condition holds.

Combining all derivations above, we have concluded the following Theorem.

Theorem 3. Assume that $\Re_{1}>0$ and $\mathfrak{R}_{0}=1$ hold. Let us denote $\tau^{*}=$ $\min \left\{\tau_{n}: n=0,1,2, ..\right\}>0$ such that $\omega\left(\tau^{*}\right)=\omega_{0}$. Then Eq. (4.2) has a simple root $z$ zero, and also its all other roots have negative real parts for $\tau \in\left[0, \tau^{*}\right)$. Therefore, $S^{*}$ is stable for $\tau \in\left[0, \tau^{*}\right)$. Moreover, system (2.1) undergoes a zero-Hopf bifurcation at $S^{*}$ when $\tau$ passes through $\tau^{*}$.

4.2. Bifurcation analysis of system (2.1) when $\tau_{1}>0$ and $\tau_{2}=0$

Under the condition $\mathfrak{R}_{0}=1$, the corresponding characteristic equation of system (2.1) is:

$$
P\left(\lambda, \tau_{1}\right) \equiv \lambda^{4}+a_{1} \lambda^{3}+a_{2} \lambda^{2}+a_{3} \lambda+e^{-\lambda \tau_{1}}\left(b_{1} \lambda^{3}+b_{2} \lambda^{2}+b_{3} \lambda\right)=0,
$$

where

$$
\begin{aligned}
& a_{1}=c+\mu_{1}+\mu_{2}+k_{2} \vartheta, \\
& a_{2}=\left(\mu_{1}+k_{2} \vartheta\right)\left(c+\mu_{2}\right), \\
& a_{3}=k_{2} \vartheta c \mu_{2}, \\
& b_{1}=\frac{\theta r_{1}\left(\mu_{1}+k_{2} \vartheta\right)}{r_{2}-\theta r_{1}}, \\
& b_{2}=\frac{\theta r_{1}}{r_{2}-\theta r_{1}}\left(c+\mu_{2}\right)\left(\mu_{1}+k_{2} \vartheta\right)+r_{1}\left(\mu_{1}+k_{2} \vartheta\right), \\
& b_{3}=r_{1}\left(\mu_{1}+k_{2} \vartheta\right)\left(c+\mu_{2}\right) .
\end{aligned}
$$


$P\left(\lambda, \tau_{1}\right)$ in Eq. (4.9) is a transcendental polynomial and has infinitely many roots. First, it is easy to see that one of its roots is zero. This is a simple root since $a_{3}$ and $b_{3}$ are positive because of the positivity of the parameters of the model.

In this section, we will determine the conditions on the parameters for the occurrence of a zero-Hopf bifurcation. To do this, we first need to show the existence of a pair of purely imaginary roots for Eq. (4.9).

Let us denote $\lambda=\eta\left(\tau_{1}\right)+i w\left(\tau_{1}\right)$. We look for a pair of purely imaginary roots such that $\lambda\left(\tau_{1}^{*}\right)=i \omega\left(\tau_{1}^{*}\right)=i \omega_{1}, \omega_{1}>0$ (without loss of generality). It is clear that $\lambda=i \omega, \omega>0$, is a root of Eq. (4.9) if

$$
(i \omega)^{3}+a_{1}(i \omega)^{2}+a_{2}(i \omega)+a_{3}+e^{-(i \omega) \tau_{1}}\left(b_{1}(i \omega)^{2}+b_{2}(i \omega)+b_{3}\right)=0
$$

Separating the real and imaginary parts, we obtain the following equations:

$$
\begin{array}{ll}
-a_{1} \omega^{2}+a_{3} & =b_{1} \omega^{2} \cos \left(\omega \tau_{1}\right)-b_{3} \cos \left(\omega \tau_{1}\right)-b_{2} \omega \sin \left(\omega \tau_{1}\right), \\
\omega^{3}-a_{2} \omega & =b_{2} \omega \cos \left(\omega \tau_{1}\right)+b_{1} \omega^{2} \sin \left(\omega \tau_{1}\right)-b_{3} \sin \left(\omega \tau_{1}\right) .
\end{array}
$$

Squaring first both sides of these equations and then adding them up, one reaches to the following equation:

$$
\omega^{6}+\left(a_{1}^{2}-2 a_{2}-b_{1}^{2}\right) \omega^{4}+\left(a_{2}^{2}-2 a_{1} a_{3}+2 b_{1} b_{3}-b_{2}^{2}\right) \omega^{2}+a_{3}^{2}-b_{3}^{2}=0
$$

Now, let us take $\omega^{2}=z$, then we can rewrite Eq. (4.11) as follows:

$$
z^{3}+\left(a_{1}^{2}-2 a_{2}-b_{1}^{2}\right) z^{2}+\left(a_{2}^{2}-2 a_{1} a_{3}+2 b_{1} b_{3}-b_{2}^{2}\right) z+a_{3}^{2}-b_{3}^{2}=0 .
$$

It is known that the expression $b_{3}^{2}-a_{3}^{2}$ is equal to the product of the roots by the Vieta's Theorem [23]. Therefore, the product of the roots of Eq. (4.12) must be positive. Then, at least one of them must be positive since Eq. (4.12) has at most three roots. Let us define the following:

$$
\Re_{2}=b_{3}^{2}-a_{3}^{2}=r_{1}\left(\mu_{1}+k_{2} \vartheta\right)\left(c+\mu_{2}\right)-k_{2} \vartheta c \mu_{2}
$$

then, assume that $\Re_{2}>0$. This guarantees that there is at least one positive root of Eq. (4.12). Following that, there exist at least one positive root o (4.11), too. Thus, the characteristic equation (4.9) has a pair of purely imaginary roots $\pm i \omega_{1}$ at $\tau_{1}^{*}$. Now, if we solve $\sin (\omega \tau)$ and $\cos (\omega \tau)$ from Eq. (4.10) simultaneously, we obtain the following equations:

$$
\begin{aligned}
& \cos (\omega \tau)=\frac{b_{2} \omega^{2}\left(\omega^{2}-a_{2}\right)+\left(b_{1} \omega^{2}-b_{3}\right)\left(a_{3}-a_{1} \omega^{2}\right)}{b_{2}^{2} \omega^{2}+\left(b_{1} \omega^{2}-b_{3}\right)^{2}}, \\
& \sin (\omega \tau)=\frac{\left(a_{2} \omega-\omega^{3}\right)\left(b_{3}-b_{1} \omega^{2}\right)-b_{2} \omega\left(a_{3}-a_{1} \omega^{2}\right)}{b_{2}^{2} \omega^{2}+\left(b_{1} \omega^{2}-b_{3}\right)^{2}} .
\end{aligned}
$$

Finally, utilizing these equations we solve $\tau_{1}^{n}$ for $n=0,1,2, \ldots$ as follows:

$$
\tau_{1}^{n}=\frac{1}{\omega} \arccos \left(\frac{b_{2} \omega^{2}\left(\omega^{2}-a_{2}\right)+\left(b_{1} \omega^{2}-b_{3}\right)\left(a_{3}-a_{1} \omega^{2}\right)}{b_{2}^{2} \omega^{2}+\left(b_{1} \omega^{2}-b_{3}\right)^{2}}\right)+\frac{2 \pi n}{\omega}
$$


Let us denote $\tau_{1}^{*}=\min \left\{\tau_{1}^{n}: n=0,1,2, ..\right\}>0$ such that $i \omega_{1}=i \omega\left(\tau_{1}^{*}\right)$ is a root of Eq. (4.9). Now, differentiating both sides of Eq. (4.9) with respect to $\tau_{1}$, one can show that the transversality condition holds.

Theorem 4. Assume that $\Re_{1}>0, \Re_{2}>0$ and $\Re_{0}=1$ hold. Let us denote $\tau_{1}^{*}=$ $\min \left\{\tau_{1}^{n}: n=0,1,2, ..\right\}>0$ such that $\omega\left(\tau_{1}^{*}\right)=\omega_{1}$. Then Eq. (4.9) has a simple root zero, and also its all other roots have negative real parts for $\tau_{1} \in\left[0, \tau_{1}^{*}\right)$. Therefore, $S^{*}$ is stable for $\tau_{1} \in\left[0, \tau_{1}^{*}\right)$. Moreover, system (2.1) undergoes a zero-Hopf bifurcation at $S^{*}$ when $\tau_{1}$ passes through $\tau_{1}^{*}$.

\subsection{Bifurcation analysis of system (2.1) when $\tau_{1}>0, \tau_{2}>0$ but $\tau_{1} \neq \tau_{2}$}

Under the condition $\Re_{0}=1$, the corresponding characteristic equation of linearized system at $S^{*}$ as follows:

$$
P\left(\lambda, \tau_{1}, \tau_{2}\right) \equiv \lambda\left(P_{0}(\lambda)+P_{1}(\lambda) e^{-\lambda \tau_{1}}+P_{2}(\lambda) e^{-\lambda \tau_{2}}\right)=0,
$$

where

$$
\begin{aligned}
& P_{0}(\lambda)=\lambda^{3}+a_{1} \lambda^{2}+a_{2} \lambda, \\
& P_{1}(\lambda)=b_{1} \lambda^{2}+b_{2} \lambda+b_{3}, \\
& P_{2}(\lambda)=c_{1} \lambda^{2}+c_{2} \lambda+c_{3} .
\end{aligned}
$$

The coefficients can be found in the following

$$
\begin{aligned}
a_{1} & =c+\mu_{1}+\mu_{2}, \\
a_{2} & =\mu_{1}\left(c+\mu_{2}\right), \\
b_{1} & =\frac{\theta r_{1}\left(\mu_{1}+k_{2} \vartheta\right)}{r_{2}-\theta r_{1}}, \\
b_{2} & =\frac{\theta r_{1}}{r_{2}-\theta r_{1}}\left(c+\mu_{2}\right)\left(\mu_{1}+k_{2} \vartheta\right)+r_{1}\left(\mu_{1}+k_{2} \vartheta\right), \\
b_{3} & =r_{1}\left(\mu_{1}+k_{2} \vartheta\right)\left(c+\mu_{2}\right), \\
c_{1} & =k_{2} \vartheta, \\
c_{2} & =k_{2} \vartheta\left(c+\mu_{2}\right), \\
c_{3} & =k_{2} \vartheta c \mu_{2} .
\end{aligned}
$$

$P\left(\lambda, \tau_{1}, \tau_{2}\right)$ in Eq. (4.15) is a transcendental polynomial and has infinitely many roots. Again, it is easy to see that one of its roots is zero. This is a simple root since $b_{3}$ and $c_{3}$ are positive because of the positivity of the parameters of the model.

In this section, we investigate the existence of the zero-Hopf bifurcation with $\tau_{1}$ in its interval of stability, regarding $\tau_{2}$ as a parameter. So, we consider the system under the previous case.

Let us denote $\lambda=\eta\left(\tau_{2}\right)+i w\left(\tau_{2}\right)$. We look for a pair of purely imaginary roots such that $\lambda\left(\tau_{2}^{*}\right)=i \omega\left(\tau_{2}^{*}\right)=i \omega_{2}, \omega_{2}>0$. Choosing $\tau_{2}$ as a parameter, we obtain that 
$\lambda=i \omega, \omega>0$, is a root of Eq. (4.15) if

$$
P\left(i \omega, \tau_{1}, \tau_{2}\right) \equiv i \omega\left(P_{0}(i \omega)+P_{1}(i \omega) e^{-i \omega \tau_{1}}+P_{2}(i \omega) e^{-i \omega \tau_{2}}\right)=0
$$

$P_{i}(i \omega)$ are given in the following:

$$
\begin{aligned}
& P_{0}(i \omega)=-a_{1} \omega^{2}+i\left(-\omega^{3}+a_{2} \omega\right), \\
& P_{1}(i \omega)=\left(b_{3}-b_{1} \omega^{2}\right)+i\left(b_{2} \omega\right), \\
& P_{2}(i \omega)=\left(c_{3}-c_{1} \omega^{2}\right)+i\left(c_{2} \omega\right) .
\end{aligned}
$$

Since $\left|e^{-i \omega \tau_{1}}\right|=1$ we have the following equations:

$$
\begin{aligned}
\left|P_{0}(i \omega)+P_{2}(i \omega) e^{-i \omega \tau_{2}}\right| & =\left|P_{1}(i \omega) e^{-i \omega \tau_{1}}\right| \\
& =\left|P_{1}(i \omega)\right|\left|e^{-i \omega \tau_{1}}\right| \\
& =\left|P_{1}(i \omega)\right|
\end{aligned}
$$

which equals to

$$
\begin{aligned}
\left|P_{0}(i \omega)+P_{2}(i \omega) e^{-i \omega \tau_{2}}\right|^{2} & =\left|P_{1}(i \omega)\right|^{2} \\
\left(P_{0}(i \omega)+P_{2}(i \omega) e^{-i \omega \tau_{2}}\right)\left(\bar{P}_{0}(i \omega)+\bar{P}_{2}(i \omega) e^{i \omega \tau_{2}}\right) & =\left|P_{1}(i \omega)\right|^{2} .
\end{aligned}
$$

After some simplifications, the last equation becomes

$$
\begin{aligned}
& \left|P_{0}(i \omega)\right|^{2}+\left|P_{2}(i \omega)\right|^{2}+2 \operatorname{Re}\left(P_{0}(i \omega) \bar{P}_{2}(i \omega)\right) \cos \left(\omega \tau_{2}\right) \\
& -2 \operatorname{Im}\left(P_{0}(i \omega) \bar{P}_{2}(i \omega)\right) \sin \left(\omega \tau_{2}\right)=\left|P_{1}(i \omega)\right|^{2} .
\end{aligned}
$$

Note that for $\omega>0, P_{0}(i \omega)=-a_{1} \omega^{2}+i\left(-\omega^{3}+a_{2} \omega\right) \neq 0$ and $P_{2}(i \omega)=\left(c_{3}-c_{1} \omega^{2}\right)+$ $i\left(c_{2} \omega\right) \neq 0$. So the folllowing inequality holds.

$$
\left[\operatorname{Re}\left(P_{0}(i \omega) \bar{P}_{2}(i \omega)\right)\right]^{2}+\left[\operatorname{Im}\left(P_{0}(i \omega) \bar{P}_{2}(i \omega)\right)\right]^{2}=\left|P_{0}(i \omega) \bar{P}_{2}(i \omega)\right|^{2}>0 .
$$

Finally, the Eqn. (4.16) can be written as

$$
\begin{aligned}
& \frac{\left|P_{1}(i \omega)\right|^{2}-\left|P_{0}(i \omega)\right|^{2}-\left|P_{2}(i \omega)\right|^{2}}{2\left|P_{0}(i \omega) \bar{P}_{2}(i \omega)\right|}=\frac{\operatorname{Re}\left(P_{0}(i \omega) \bar{P}_{2}(i \omega)\right)}{\left|P_{0}(i \omega) \bar{P}_{2}(i \omega)\right|} \cos \left(\omega \tau_{2}\right) \\
& -\frac{\operatorname{Im}\left(P_{0}(i \omega) \bar{P}_{2}(i \omega)\right)}{\left|P_{0}(i \omega) \bar{P}_{2}(i \omega)\right|} \sin \left(\omega \tau_{2}\right) .
\end{aligned}
$$

On the other hand, one can show that there is a continous $\phi(\omega)$ function such that:

$$
\cos (\phi(\omega))=\frac{\operatorname{Re}\left(P_{0}(i \omega) \bar{P}_{2}(i \omega)\right)}{\left|P_{0}(i \omega) \bar{P}_{2}(i \omega)\right|} \text { and } \sin (\phi(\omega))=\frac{\operatorname{Im}\left(P_{0}(i \omega) \bar{P}_{2}(i \omega)\right)}{\left|P_{0}(i \omega) \bar{P}_{2}(i \omega)\right|}
$$

holds. Therefore, we have the following:

and so,

$$
\left|\frac{\left|P_{1}(i \omega)\right|^{2}-\left|P_{0}(i \omega)\right|^{2}-\left|P_{2}(i \omega)\right|^{2}}{2\left|P_{0}(i \omega) \bar{P}_{2}(i \omega)\right|}\right|=\left|\cos \left(\phi(\omega)+\omega \tau_{2}\right)\right| \leq 1
$$

$$
\left.|| P_{1}(i \omega)\right|^{2}-\left|P_{0}(i \omega)\right|^{2}-\left|P_{2}(i \omega)\right|^{2}|\leq 2| P_{0}(i \omega) \bar{P}_{2}(i \omega) \mid .
$$


Finally, if we define

$$
\Omega=\left\{\omega>0:\left.|| P_{1}(i \omega)\right|^{2}-\left|P_{0}(i \omega)\right|^{2}-\left|P_{2}(i \omega)\right|^{2}|\leq 2| P_{0}(i \omega) \bar{P}_{2}(i \omega) \mid\right\}
$$

then we have the following lemma:

Lemma 3. Let $\Re_{2}=r_{1}\left(\mu_{1}+k_{2} v\right)\left(c+\mu_{2}\right)-k_{2} v c \mu_{2}$. If $\Re_{2}>0$ then the set $\Omega$ is not empty.

The above lemma means that for $n=0,1,2, \ldots$ and $\omega \in \Omega$ the characteristic equation has purely imaginary roots when

$$
\tau_{2}^{n}=\frac{\psi\left(\omega_{2}\right)-\phi\left(\omega_{2}\right)+2 \pi n}{\omega_{2}}
$$

where

$$
\cos \left(\psi\left(\omega_{2}\right)\right)=\frac{\left|P_{1}(i \omega)\right|^{2}-\left|P_{0}(i \omega)\right|^{2}-\left|P_{2}(i \omega)\right|^{2}}{2\left|P_{0}(i \omega) \bar{P}_{2}(i \omega)\right|} .
$$

On the other hand, we can write the characteristic equation as the form of

$$
P(\lambda)+Q(\lambda) e^{-\lambda \tau_{2}}=0
$$

where

$$
\begin{aligned}
& P(\lambda)=P_{0}(\lambda)+P_{1}(\lambda) e^{-\lambda \tau_{1}}, \\
& Q(\lambda)=P_{2}(\lambda) .
\end{aligned}
$$

So, let's define

$$
F(\omega)=|P(i \omega)|^{2}-|Q(i \omega)|^{2} .
$$

After some calculations, one can get the following inequality

$$
\begin{aligned}
w_{2} F^{\prime}\left(w_{2}\right) & =A w_{2}^{6}+B w_{2}^{5}+C w_{2}^{4}+D w_{2}^{3}+E w_{2}^{2}+F w_{2}+G \\
& =w_{2}^{4}\left(A w_{2}^{2}+C\right)+w_{2}^{3}\left(B w_{2}^{2}+D\right)+w_{2}\left(E w_{2}+F\right)+G
\end{aligned}
$$

where the coefficients are

$$
\begin{aligned}
A= & \left(2-2 b_{1} \sin \omega_{2} T_{1}\right) \\
B= & \left(T_{1}\left(2 a_{1} b_{1}+2 b_{2}\right) \sin \omega_{2} T_{1}-2 b_{1} \sin \omega_{2} T_{1}\right) \\
C= & \left(T_{1} 2 b_{3} \cos \omega_{2} T_{1}-T_{1} 2 a_{1} b_{2} \cos \omega_{2} T_{1}+T_{1} 2 a_{2} b_{1} \cos \omega_{2} T_{1}\right) \\
D= & \left(-T_{1} 2 a_{2} b_{2} \sin \omega_{2} T_{1}+T_{1} 2 a_{1} b_{3} \sin \omega_{2} T_{1}\right) \\
E= & \left(-T_{1} 2 a_{2} b_{3} \cos \omega_{2} T_{1}-2 a_{2}^{2}-4 a_{2} b_{2} \cos \omega_{2} T_{1}-2 b_{2}^{2}-2 c_{2}^{2}+4 b_{1} b_{3}\right. \\
& \left.-4 a_{1} b_{3} \cos \omega_{2} T_{1}-4 c_{1} c_{3}\right) \\
F= & \left(6 a_{2} b_{3} \sin \omega_{2} T_{1}\right) \\
G= & 4\left(c_{3}^{2}-b_{3}^{2}\right)
\end{aligned}
$$

Under the following conditions 
H1): $m_{1}=\frac{-C}{A} \cdot m_{1} \geq 0$ and $A \neq 0$ and $\omega_{2}<\sqrt{m_{1}}$,

H2): $m_{2}=\frac{-D}{B} . m_{2} \geq 0$ and $B \neq 0$ and $\omega_{2}<\sqrt{m_{2}}$,

H3): $m_{3}=\frac{-F}{E} . E \neq 0$ and $\omega_{2}<m_{3}$,

it is clear that $F^{\prime}\left(w_{2}\right)<0$ holds. This inequality also means that the transversality condition holds. Hence, we have the following result.

Theorem 5. Assume that the conditions $(H 1),(H 2)$ and $(H 3)$ defined above are satisfied and also $\Re_{0}=1, \Re_{1}>0$ and $\Re_{2}>0$ hold. Let us denote $\tau_{2}^{*}=$ $\min \left\{\tau_{2}^{n}: n=0,1,2, ..\right\}>0$ such that $\omega\left(\tau_{2}^{*}\right)=\omega_{2}$. Then,

(1) Eq. (4.15) has a simple root zero, and also its all other roots have negative real parts for $\tau_{1} \in\left[0, \tau_{1}^{*}\right)$ and $\tau_{2} \in\left[0, \tau_{2}^{*}\right)$ such that $i \omega=i \omega\left(\tau_{2}^{*}\right), \tau_{2}^{*}>0$. Therefore, $S^{*}$ is stable for $\tau_{1} \in\left[0, \tau_{1}^{*}\right)$ and $\tau_{2} \in\left[0, \tau_{2}^{*}\right)$.

(2) For $\tau_{1} \in\left[0, \tau_{1}^{*}\right)$, system (2.1) undergoes a zero-Hopf bifurcation at $S^{*}$ when $\tau_{2}$ passes through $\tau_{2}^{*}$.

\section{NUMERICAL SIMULATIONS}

In this section, we perform numerical simulations that support the analytic results proved in former sections. For each simulation, we use either the ODE package (ode45) or the DDE package (dde23) in MATLAB. To illustrate the theoretical results, we study numerically the dynamics of system (2.1) with the parameter values which are chosen from Table 1 below. Choosing parameter values characteristic of the in vivo situation is difficult; many of the parameters in our model have not been measured, or, if measurements have been attempted, they may not be as accurate as we need for quantitative predictions [25]. Thefore we choose these parameters for the reason that either they are used in other models describing a similar phenomenon or they are based on experimental data in the corresponding references.

\subsection{Numerical simulations when $\tau_{1}=\tau_{2}=0$}

First, we use the following parameter values for simulations: $r_{1}=0.3, k_{1}=0.001$, $r_{2}=0.05, \mu_{1}=0.03, \theta=0.1, k_{2}=2.4 \times 10^{-5}, c=0.3, N=275, \delta=0.3$ and $\mu_{2}=$ 2.1. With respect to these parameters, the corresponding basic reproduction number and the disease-free steady state are $\mathfrak{R}_{0}=0.942857$ and $S_{0}=\left(T_{0}, E_{0}, I_{0}, V_{0}\right)=$ $(450,300,0,0)$, respectively. Figure 1 illustrates the numerical solutions of the ODE model (3.1) for different initial values. It shows that as stated in Theorem 1 and Theorem 2, the disease-free steady state is globally asymptotically stable. These figures underline the ability of the immune system to eliminate the infection and to prevent more tumour cell growth. So, the immune system eradicates virus perfectly but has low persistence of the tumour. 
TABLE 1. The range of parameter values with corresponding references

\begin{tabular}{cllc}
\hline Parameters & Range Values & Unit & References \\
\hline$r_{1}$ & $0.05-0.5$ & day $^{-1}$ & {$[28]$} \\
$k_{1}$ & $10^{-3}-10^{-5}$ & $\mathrm{~mm}^{3}$ day $^{-1}$ & {$[25]$} \\
$r_{2}$ & $0-0.05$ & day $^{-1}$ & {$[25]$} \\
$\mu_{1}$ & 0.03 & $\mathrm{~mm}^{3}$ day $^{-1}$ & {$[11]$} \\
$\theta$ & 0.1 & day $^{-1}$ & {$[29]$} \\
$k_{2}$ & $2.4 \times 10^{-5}$ & $\mathrm{~mm}^{3}$ day $^{-1}$ & {$[32]$} \\
$c$ & 0.3 & day $^{-1}$ & {$[29]$} \\
$N$ & $100-2000$ & & {$[11]$} \\
$\delta$ & $0.3-0.7$ & day $^{-1}$ & {$[26]$} \\
$\mu_{2}$ & $2.1-3.8$ & day $^{-1}$ & {$[29]$}
\end{tabular}
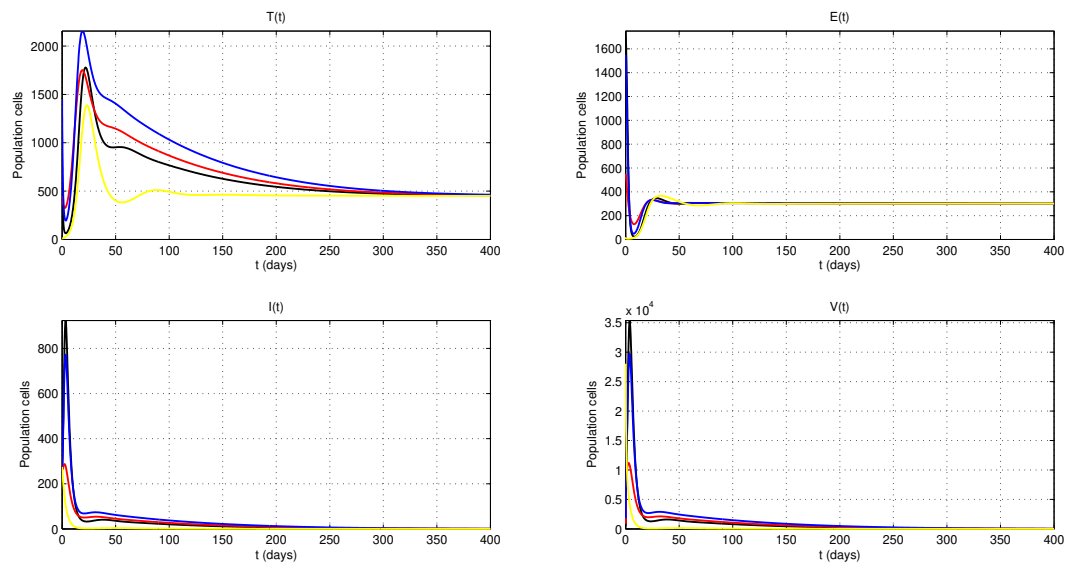

FIGURE 1. The numerical solutions of the ODE model (2.1) with different initial values. Each color represents a different solution with different initial values.

Second, Figure 3 presents the behaviour of solutions of system (3.1) for the parameter values: $r_{1}=0.1, k_{1}=0.0002, r_{2}=0.04, \mu_{1}=0.03, \theta=0.1, k_{2}=2.4 \times 10^{-5}$, $c=0.3 N=275, \delta=0.3$ and $\mu_{2}=2.1$. The corresponding basic reproduction number is equal to $\Re_{0}=1.571429$, and the disease free steady state is $S_{0}=\left(T_{0}, E_{0}, I_{0}, V_{0}\right)=$ $(500,500,0,0)$ for this case. The uncontrolled growth of the tumour cells can be seen in Figure 3. The weakness caused by HIV may advance to uncontrolled tumour growth and spread in this case.

Third, we now solve the system with the parameter values that are $r_{1}=0.1, k_{1}=$ $0.0003, r_{2}=0.03, \mu_{1}=0.03, \theta=0.1, k_{2}=2.4 \times 10^{-5}, c=0.3, N=275, \delta=$ 
0.3 and $\mu_{2}=2.2$. The corresponding basic reproduction number for this case is equal to $\Re_{0}=1$, so the endemic steady state is $S^{*}=(540,333.333,2.66,100)$. The coexistence of the tumour cells, the infected cells, and the virus can be seen from Figure 2 which illustrates the local stability of the disease-free steady state proven in Theorem 1. This means that the underlying HIV related tumour develops in the HIV infected individual because of the existence of virus; the tumour can escape from the surveillance of the immune system. As it can be seen from the simulations in Figure 2 , the steady state could not be globally stable which was underlined by the remark in the former section.
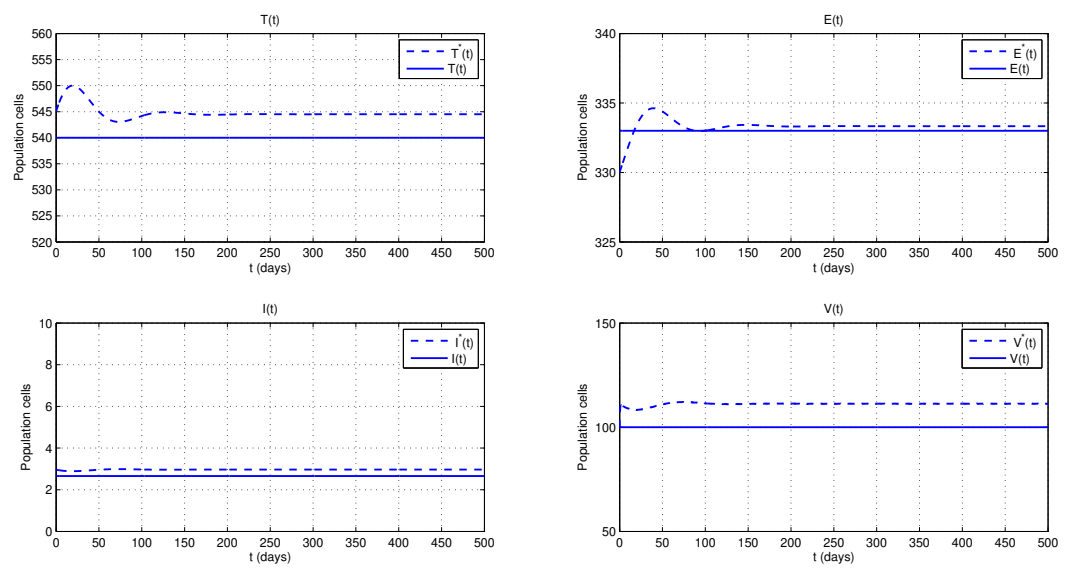

FIGURE 2. The numerical solutions of system (2.1) with initial values $\left(T_{0}, E_{0}, I_{0}, V_{0}\right)=(250,750,275,250)$. The trajectories of the system are close enough to the steady state of the system, which is denoted by the solid line, however, not approach to it.

\subsection{Numerical simulations when $\tau_{1}=\tau_{2}=\tau$}

We numerically solve the DDE model (2.1) with the parameter values: $r_{1}=0.3$, $k_{1}=0.0032, r_{2}=0.05, \mu_{1}=0.03, \theta=0.1, k_{2}=2.4 \times 10^{-5}, c=0.3, N=400, \delta=0.7$, and $\mu_{2}=2.1$. Figures 4,5 and 6 present the numerical solutions of the DDE system (2.1) when $\tau=1.95, \tau=1.962$ and $\tau=1.97$, respectively. These figures show that $\tau$ plays a crucial role in the oscillations of the solutions around steady state. Clearly, delay causes the appearance of oscillations, and affects the stability of steady state.

Also, Figures 5 and 7 show the periodic solutions arising as the bifurcation parameter $\tau$ passes through the critical value $\tau^{*} \approx 1.962$. Here, one observes the occurrence of limit cycles due to the zero-Hopf bifurcation at $\tau=1.95$. Figure 6 displays that the system becomes unstable for the value of $\tau$ which is grater than $\tau^{*}$. 

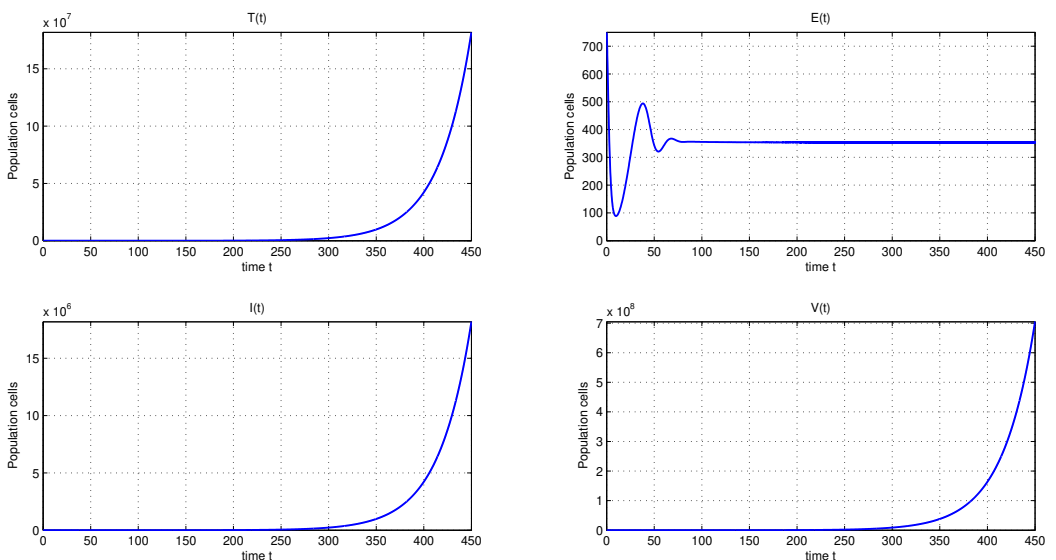

FIGURE 3. The numerical solutions of system (2.1) with initial values $\left(T_{0}, E_{0}, I_{0}, V_{0}\right)=(250,750,275,250)$. Note that the trajectories of the system move away.
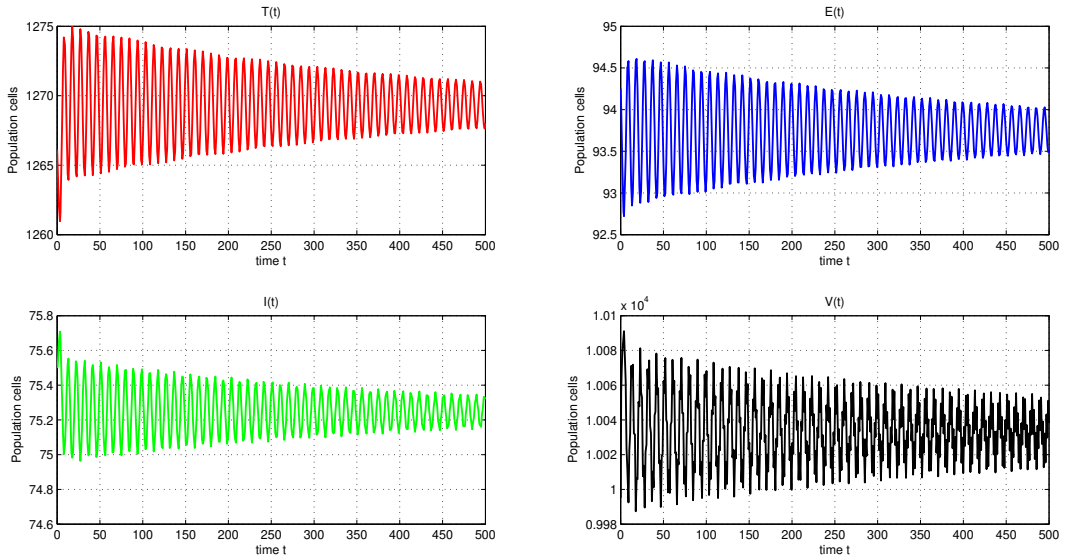

FIGURE 4. The numerical solutions of the DDE model (2.1) for $\tau=$ $1.95<\tau^{*} \approx 1.962$. Simulations present stability.

\subsection{Numerical simulations when $\tau_{1}>0, \tau_{2}>0, \tau_{1} \neq \tau_{2}$}

We numerically solve the DDE model (2.1) with the parameter values: $r_{1}=0.3$, $k_{1}=0.0032, r_{2}=0.05, \mu_{1}=0.03, \theta=0.1, k_{2}=2.4 \times 10^{-5}, c=0.3, N=400$, $\delta=0.7$, and $\mu_{2}=2.1$. Figures 8 presents the numerical solutions of the DDE system 

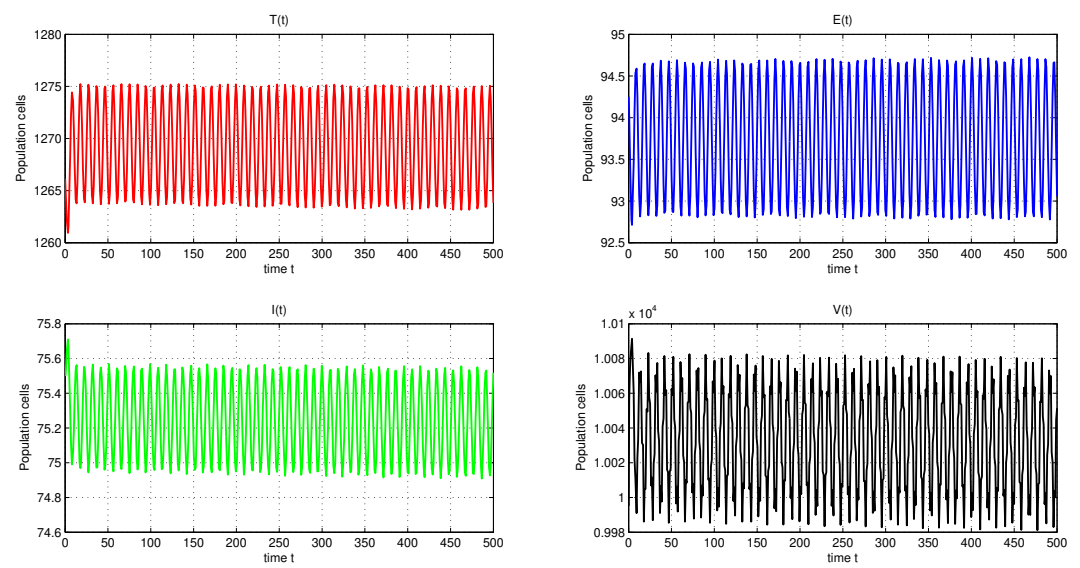

FIGURE 5. The numerical solutions of the DDE model (2.1) for $\tau=$ $\tau^{*} \approx 1.962$. They present the cyclic behaviour.
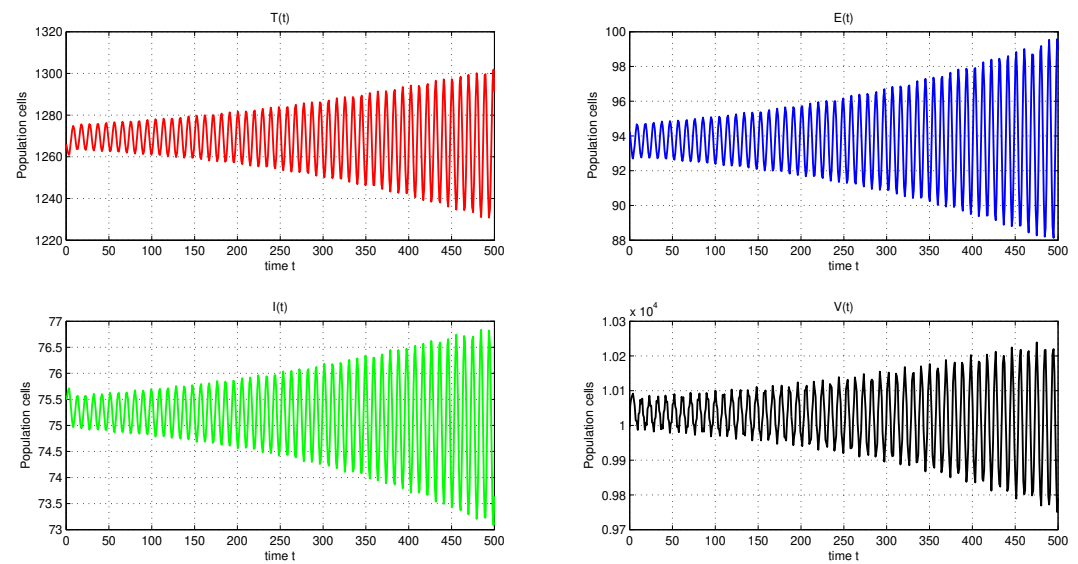

FIGURE 6. The numerical solutions of the DDE model (2.1) for $\tau=$ $1.97>\tau^{*} \approx 1.962$. Simulations show unstability.

(2.1) when $\tau_{1}=1.8<\tau_{1}^{*}$ and $\tau_{2}=2.3415$. These figures show that $\tau_{2}$ plays a crucial role in the oscillations of the solutions around steady state. Clearly, delay causes the appearance of oscillations, and affects the stability of steady state.

Also, Figures 8 and 9 show the periodic solutions arising as the bifurcation parameter $\tau$ passes through the critical value $\tau_{2}^{*} \approx 2.3415$. Here, one observes the occurrence of limit cycles due to the zero-Hopf bifurcation at $\tau_{2}=2.3415$. 

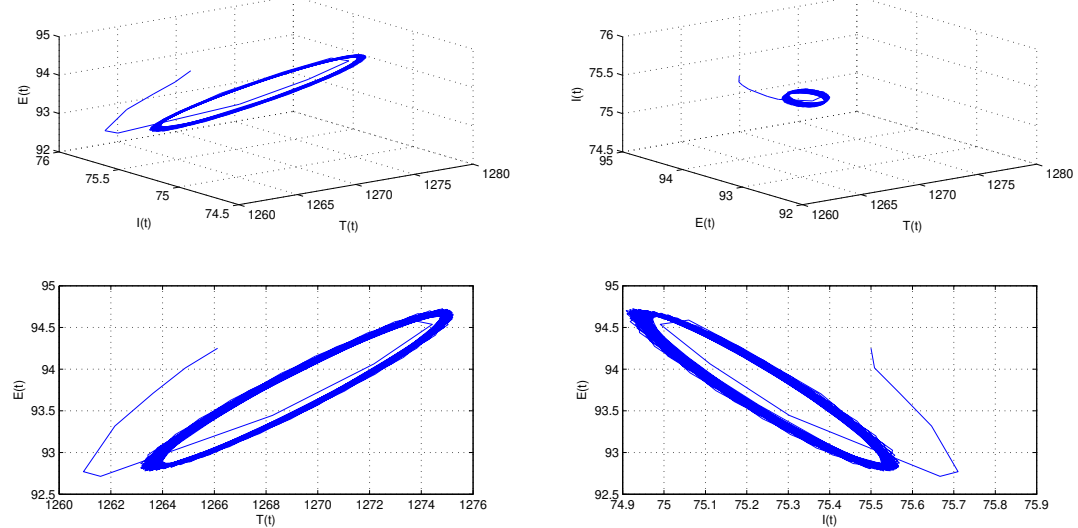

FIGURE 7. The numerical solutions of the DDE model (2.1) for $\tau=$ $\tau^{*} \approx 1.962$.
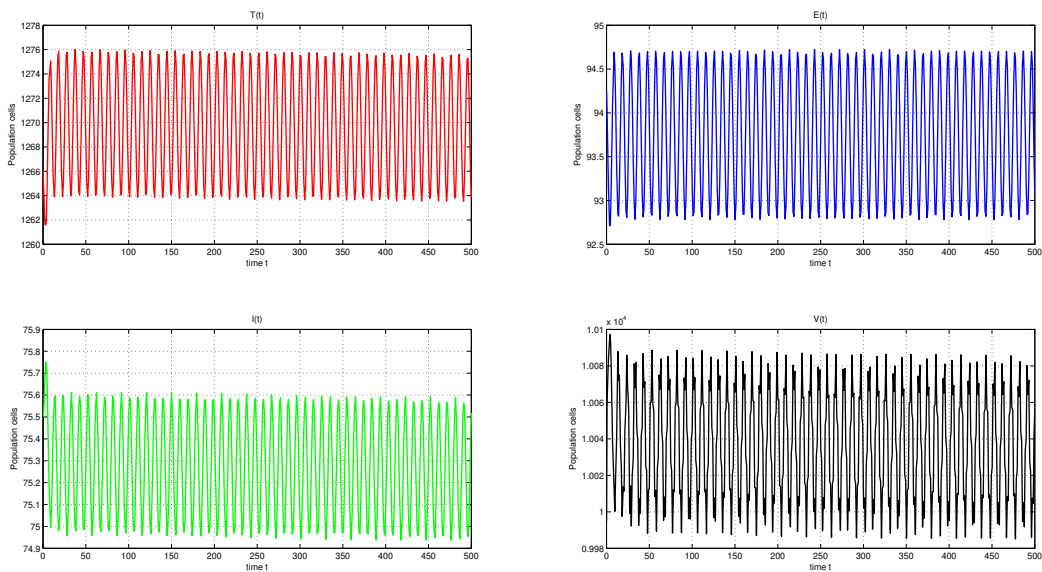

FIGURE 8. The numerical solutions of the DDE model (2.1) for $\tau_{1}=$ $1.8<\tau_{1}^{*}$ and $\tau_{2} \approx \tau_{2}^{*}=2.3415$. They present the cyclic behaviour.

\section{DisCusSION}

HIV infected individuals suffer several types tumours during their infection, which creates a burden on the treatment. For this reason, it is very crucial to understand the relationship between HIV infection and certain tumours. In this paper, we have shown how HIV affects the immune system's ability to fight tumours. Since HIV is 

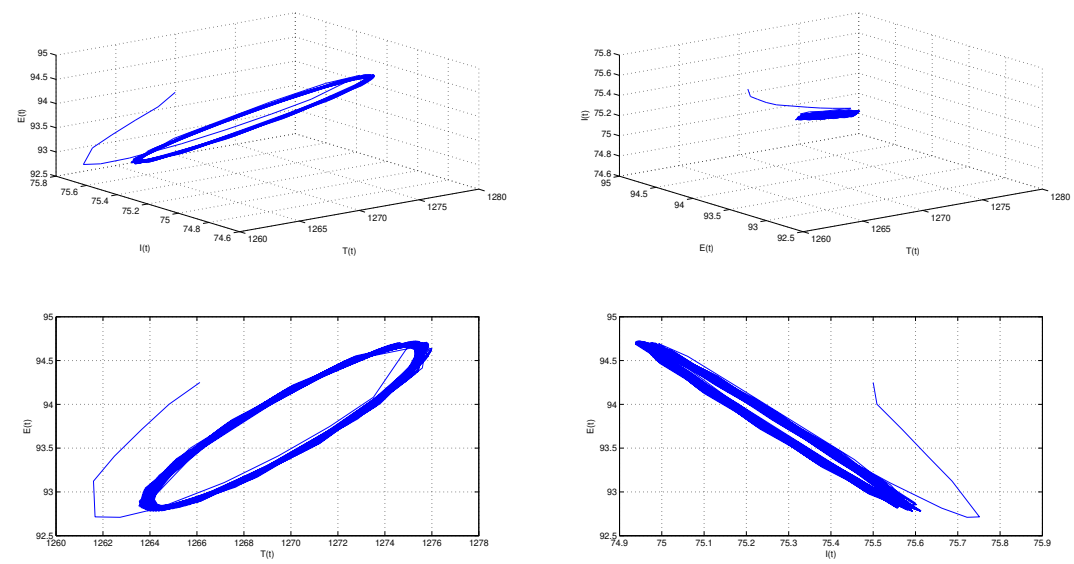

FIGURE 9. The numerical solutions of the DDE model (2.1) for $\tau_{1}=$ $1.8<\tau_{1}^{*}$ and $\tau_{2} \approx \tau_{2}^{*}=2.3415$.

present in vivo, the surveillance of the immune system is very weak. Accordingly, the growth of tumour is uncontrolled.

The model we introduced involves four components: tumour cells, uninfected helper T-cells, infected helper T-cells and free virus. Under the condition $\Re_{1}>0$, system (2.1) has two non-negative steady states. This condition means that the ratio of the rate of growth of the T-helper cells (triggered by tumour cells) with respect to the rate of growth of tumour cells is larger than the percentage of the helper cell loss due to killing tumour cells. First, the existence and positiveness of the solutions of the model without delay are studied. By utilizing the Routh-Hurwitz criteria, we have determined the conditions on the parameters for the stability of steady states of the model. When the condition $\Re_{0}<1$, we have proved that the non-infected steady state is both locally and globally asymptotically stable. This means that the ability of the immune system to eliminate the virus is strong enough but also there is low persistence of tumour. Also, we obtained that uncontrolled tumour growth and spread can be possible because of the exhaustion of the immune system response caused by infection. On the other hand, the infected steady state is L-stable. This biologically means that tumour can escape from surveillance of the immune system.

Second, the zero-Hopf bifurcation of the delay differential equation (DDE) model is studied. We study the effects of two discrete time delays on the stability of the endemically infected equilibrium point. We determine the conditions on parameters at which the system undergoes a zero-Hopf bifurcation. The time lags in the DDE model describe the time needed by the helper T-cells to find (or recognize) tumour cells and virus. Choosing one of the delay terms as a bifurcation parameter and fixing 
the other, we have shown that a zero-Hopf bifurcation occurs as the bifurcation parameter passes through a critical value. In other words, the stability of the steady state $S^{*}$ changes from stable to unstable, and periodic solutions with increasing periods arise. For the larger values of the bifurcation parameter, solutions become unstable. The performed numerical simulations support and extend our analytical results.

The results concluded underline that as the immune system gets weaker, it becomes difficult to keep up of the T-helper cells which leads to the compute exhaustion of tumour cells.

\section{REFERENCES}

[1] J. A. Adam and N. Bellomo, A survey of models for tumor-immune system dynamics. Springer Science \& Business Media, 2012.

[2] B. Alberts, D. Bray, K. Hopkin, A. D. Johnson, J. Lewis, M. Raff, K. Roberts, and P. Walter, Essential cell biology. Garland Science, 2013.

[3] L. J. Allen, Introduction to mathematical biology. Pearson/Prentice Hall, 2007.

[4] C. Bellan, G. D. Falco, S. Lazzi, and L. Leoncini, "Pathologic aspects of aids malignancies," Oncogene, vol. 22, no. 42, pp. 6639-6645, 2003, doi: 10.1038/sj.onc.1206815.

[5] M. Bodnar, U. Foryś, and Z. Szymańska, "Model of aids-related tumour with time delay," Applicationes Mathematicae, vol. 36, no. 3, pp. 263-278, 2009, doi: 10.4064/am36-3-2.

[6] C. Boshoff and R. Weiss, "Aids-related malignancies," Nature Reviews Cancer, vol. 2, no. 5, pp. 373-382, 2002, doi: 10.1038/nrc797.

[7] F. M. Burnet, "Immunological surveillance in neoplasia," Immunological Reviews, vol. 7, no. 1, pp. 3-25, 1971, doi: 10.1111/j.1600-065x.1971.tb00461.x.

[8] K. C. Carroll, J. Butel, and S. Morse, Jawetz Melnick and Adelbergs Medical Microbiology, 27th ed. McGraw-Hill Education, 2015.

[9] M. P. Cranage, A. M. Whatmore, S. A. Sharpe, N. Cook, N. Polyanskaya, S. Leech, J. D. Smith, E. W. Rud, M. J. Dennis, and G. A. Hall, "Macaques infected with live attenuated sivmac are protected against superinfection via the rectal mucosa," Virology, vol. 229, no. 1, pp. 143-54, Mar 1997, doi: 10.1006/viro.1996.8419.

[10] K. A. Crandall, The evolution of HIV. JHU Press, 1999.

[11] R. V. Culshaw and S. Ruan, "A delay-differential equation model of hiv infection of cd4+ tcells," Mathematical biosciences, vol. 165, no. 1, pp. 27-39, May 2000, doi: 10.1016/s00255564(00)00006-7.

[12] U. Foryś and J. Poleszczuk, "A delay-differential equation model of hiv related cancer-immune system dynamics." Mathematical biosciences and engineering : MBE, vol. 8, no. 2, pp. 627-41, Apr 2011, doi: 10.1080/08898480.2013.804688.

[13] M. Gerloni and M. Zanetti, "Cd4 t cells in tumor immunity," vol. 27, pp. 37-48, 2005, doi: 10.1007/s00281-004-0193-z.

[14] K. Hattaf, N. Yousfi, and A. Tridane, "Stability analysis of a virus dynamics model with general incidence rate and two delays," Canadian Applied Mathematics Quarterly, vol. 221, pp. 514-521, 12 2013, doi: 10.1016/j.amc.2013.07.005.

[15] D. Kirschner, "Using mathematics to understand hiv immune dynamics," Notices of the AMS, vol. 43, no. 2, pp. 191-202, 1996, doi: 10.1006/tpbi.1998.1382.

[16] D. Kirschner and J. C. Panetta, "Modeling immunotherapy of the tumor - immune interaction," Journal of mathematical biology, vol. 37, no. 3, pp. 235-52, Sep 1998, doi: $10.1007 / \mathrm{s} 002850050127$. 
[17] D. Klatzmann, F. Barre-Sinoussi, M. Nugeyre, C. Danquet, E. Vilmer, C. Griscelli, F. BrunVeziret, C. Rouzioux, J. Gluckman, J. Chermann, and al. et, "Selective tropism of lymphadenopathy associated virus (lav) for helper-inducer t lymphocytes," Science, vol. 225, no. 4657, pp. 59-63, Jul 1984, doi: 10.1126/science.6328660.

[18] D. Klatzmann, E. Champagne, S. Chamaret, J. Gruest, D. Guetard, T. Hercend, J.-C. Gluckman, and L. Montagnier, "T-lymphocyte t 4 molecule behaves as the receptor for human retrovirus lav," Nature, vol. 312, no. 5996, pp. 767-768, 1984, doi: 10.1038/312767a0.

[19] V. A. Kuznetsov, I. A. Makalkin, M. A. Taylor, and A. S. Perelson, "Nonlinear dynamics of immunogenic tumors: Parameter estimation and global bifurcation analysis," Bulletin of mathematical biology, vol. 56, no. 2, pp. 295-321, 1994, doi: 10.1007/bf02460644.

[20] J. A. Levy, HIV and the pathogenesis of AIDS. American Society for Microbiology, 1994.

[21] J. Lou and T. Ruggeri, "A time delay model about aids-related cancer: equilibria, cycles and chaotic behavior," Ricerche di Matematica, vol. 56, pp. 195-208, 12 2007, doi: 10.1007/s11587007-0013-6.

[22] J. Lou, T. Ruggeri, and C. Tebaldi, "Modeling cancer in hiv-1 infected individuals: equilibria, cycles and chaotic behavior." Mathematical biosciences and engineering : MBE, vol. 3, no. 2, pp. 313-24, Apr 2006.

[23] H. Michiel, Encyclopedia of Mathematics. Springer, 2001.

[24] P. R. Murray, K. S. Rosenthal, and M. A. Pfaller, Medical microbiology. Elsevier Health Sciences, 2015.

[25] A. S. Perelson, D. E. Kirschner, and R. D. Boer, "Dynamics of hiv infection of cd4+ t cells," Mathematical biosciences, vol. 114, no. 1, pp. 81-125, Mar 1993, doi: 10.1016/0025-5564(93)90043-a.

[26] A. S. Perelson and P. W. Nelson, "Mathematical analysis of HIV-1 dynamics in vivo," SIAM Review, vol. 41, no. 1, pp. 3-44, 1999, doi: 10.1137/s0036144598335107.

[27] L. Preziosi, Cancer modelling and simulation. CRC Press, 2003.

[28] A. Qi and Y. Du, "Nonlinear models in immunity," Shanghai Scientific and Technological Education Publishing House, pp. 1-156, 1998.

[29] F. A. Rihan and D. H. A. Rahman, "Delay differential model for tumour-immune dynamics with hiv infection of cd4+t-cells," International Journal of Computer Mathematics, vol. 90, no. 3, pp. 594-614, 2013, doi: 10.1080/00207160.2012.726354.

[30] J. C. Sherris and K. J. Ryan, Medical microbiology: an introduction to infectious diseases. Elsevier Publishing Company, 1984.

[31] Z. Shuai and P. van den Driessche, "Global stability of infectious disease models using lyapunov functions," SIAM Journal on Applied Mathematics, vol. 73, no. 4, pp. 1513-1532, 2013, doi: $10.1137 / 120876642$.

[32] J. L. Spouge, R. I. Shrager, and D. S. Dimitrov, "Hiv-1 infection kinetics in tissue cultures," Mathematical biosciences, vol. 138, no. 1, pp. 1-22, Nov 1996, doi: 10.1016/s0025-5564(96)00064-8.

[33] D. J. Straus, "Hiv-associated lymphomas," Current oncology reports, vol. 3, no. 3, pp. 260-265, 2001, doi: 10.1007/s11912-001-0059-7.

[34] P. van den Driessche and J. Watmough, "Further notes on the basic reproduction number," pp. 159-178, 2008, doi: 10.1007/978-3-540-78911-6.

[35] T. E. Wheldon, Mathematical models in cancer research. Taylor \& Francis, 1988.

[36] C. Wood and W. Harrington, "Aids and associated malignancies," Cell research, vol. 15, no. 11, p. 947, 2005, doi: 10.1038/sj.cr.7290372. 
Authors' addresses

\section{Gamzegul Karahisarli}

TOBB University of Economics and Technology, Department of Mathematics, Ankara, Turkey

E-mail address: ggaydin@etu.edu.tr

Huseyin Merdan

TOBB University of Economics and Technology, Department of Mathematics, Ankara, Turkey

E-mail address: merdandetu.edu.tr

Abdessamad Tridane

United Arab Emirates University, Department of Mathematical Science, Al Ain, Abu Dhabi, UAE

E-mail address: a-tridane@uaeu.ac.ae 